\title{
Plasmonic Metasurfaces with Conjugated Polymers for Flexible Electronic Paper in Color
}

Kunli Xiong, Gustav Emilsson, Ali Maziz, Xinxin Yang, Lei Shao, Edwin Jager and Andreas

B. Dahlin

Journal Article

Tweet

N.B.: When citing this work, cite the original article.

Original Publication:

Kunli Xiong, Gustav Emilsson, Ali Maziz, Xinxin Yang, Lei Shao, Edwin Jager and Andreas B. Dahlin, Plasmonic Metasurfaces with Conjugated Polymers for Flexible Electronic Paper in Color, Advanced Materials, 2016. 28(45), pp.9956-9960.

http://dx.doi.org/10.1002/adma.201603358

Copyright: Wiley: 12 months

http://eu.wiley.com/WileyCDA/

Postprint available at: Linköping University Electronic Press

http://urn.kb.se/resolve?urn=urn:nbn:se:liu:diva-134782

I.v UNNGERSIIET 
DOI: 10.1002/adma.201603358

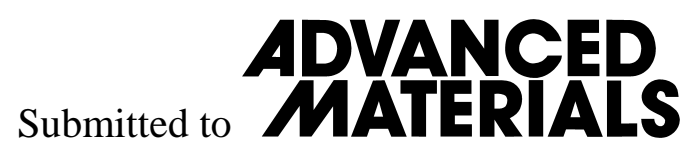

Article type: Communication

Plasmonic Metasurfaces with Conjugated Polymers for Flexible Electronic Paper in Color

Kunli Xiong, Gustav Emilsson, Ali Maziz, Xinxin Yang, Lei Shao, Edwin W. H. Jager and Andreas B. Dahlin.*

K. Xiong, G. Emilsson, X. Yang, Dr L. Shao, Dr A. B. Dahlin*

Dept. of Physics, Chalmers University of Technology, 41296 Göteborg, Sweden

* Corresponding author: adahlin@chalmers.se

Dr A. Maziz, Dr E. W. H. Jager

Dept. of Physics, Chemistry and Biology (IFM), Linköping University, 58183 Linköping, Sweden

Keywords: plasmons, metasurfaces, conjugated polymers, electrochromism, electronic paper 


\section{Submitted to

Plasmonic nanostructures have been used to generate colors for thousands of years. ${ }^{[1]}$

Recently, metallic nanostructures with fine-tuned dimensions have been used to "print” stable color images ${ }^{[2-4]}$ with extremely high lateral resolution. ${ }^{[5-7]}$ Implementing electrochromic switching with such nanostructures opens up for new display technologies.$^{[8-11]}$ Reflective (paper-like) displays are particularly interesting since they provide lower energy consumption and comfortable viewing under ambient light, ${ }^{[12]}$ typically using electrophoretic ink ${ }^{[13]}$ (e.g. the Kindle ${ }^{\mathrm{TM}}$ e-reader). Unfortunately, technologies providing reflective displays in color remain strongly limited. Conjugated polymers are interesting for this purpose, but their electrochromic activity alone generally provides low contrast. ${ }^{[14]}$ In combination with plasmonic nanostructures the tunable absorption of the polymers can be used to regulate the optical activity. ${ }^{[10,15,16]}$ However, research to date has focused on nanoparticles and only modulation of transmission. There has been no demonstration of a plasmonic nanostructure providing reflective colors that can be electrically modulated. Further, for applications in display technologies the electrochromic material must be prepared over very large areas, which excludes the use of electron beam ${ }^{[4]}$ or focused ion beam ${ }^{[10]}$ lithography. Preferably, the material should also be ultrathin and flexible ${ }^{[12]}$ making the final display foldable and less fragile, which cannot be achieved with approaches based on liquid crystals. ${ }^{[11]}$ In this work we present the first material which fulfils all criteria to work as electronic paper in full color with good potential to realize flexible screens. The electrically controllable optical absorption of a conducting polymer is used to modulate the reflected light from ultrathin nanostructured plasmonic metasurfaces. ${ }^{[17]}$ Our method provides high polarization-independent resonant reflection ( $>90 \%$ in air), good contrast (30-50\%), fast response times (ms regime), ultralow power consumption $\left(<0.5 \mathrm{mWcm}^{-2}\right)$ and long-term stability. We also show that plasmonic metasurfaces containing pixels of the primary colors red-green-blue (RGB) give the same reflectivity and contrast as ink produced by an ordinary printer. Finally, we show how the 
Submitted to

\section{ADVXNCEP}

RGB pixels can produce secondary colors and display-size images which can be switched on/off.

Our plasmonic metasurfaces contains three solid films (Figure 1a). During fabrication, a 150 nm silver film was first deposited on the substrate to provide a high base reflection. The subsequent alumina spacer layer tuned the reflective color by Fabry-Pérot interference. ${ }^{[6]}$ Next, short-range ordered $150 \mathrm{~nm}$ nanoholes in a $20 \mathrm{~nm}$ gold film (Figure 1b) were prepared on alumina by colloidal self-assembly and tape stripping. ${ }^{[18]}$ The fabrication consisted of parallel processing steps ${ }^{[19]}$ compatible with large areas and plastic supports, which made the material flexible by hand (Figure 1c). We generated a color palette by varying the alumina thickness ${ }^{[20]}$ from 40 to $95 \mathrm{~nm}$ and found that the primary colors red, green and blue (RGB samples, Figure 1d) corresponded to an alumina thickness of $48 \mathrm{~nm}, 93 \mathrm{~nm}$ and $83 \mathrm{~nm}$ respectively. The high resonant reflectivity (Figure 1e) confirmed the clear colors of the plasmonic metasurfaces.

The gold film is necessary to create coloration because the absorption of silver is very low in the visible ${ }^{[21]}$ and since there is no transmission through the thick mirror layer all visible wavelengths would then be reflected. Further, the nanohole array in the thin gold film enhances the coloration since it enables coupling to surface plasmons ${ }^{[22,23]}$ and provides strong resonant scattering. ${ }^{[18]}$ Although we could also generate colors by only a gold film without holes on the silver and alumina layers, such thin film multilayers do not support plasmon excitation under ordinary illumination and cannot scatter light. This limited the possibility to tune the reflection spectrum and resulted in more diffuse colors since only absorption $^{[21]}$ (and not scattering) could contribute (Figure 1c). By dark-field illumination we verified that the three RGB structures with nanoholes scattered their complementary colors at high angles (Figure S1, Supporting Information). We also analyzed the reflectivity for 


\section{Submitted to \\ ADVANCEP}

increasing incident angle (Figure S2, Supporting Information) and found that the viewing

angle could be up to approximately $60^{\circ}$ with correct color appearance.

To switch the colors on and off, the tuneable optical absorption of conjugated polymers was utilized. ${ }^{[24]}$ Doped polypyrrole films ${ }^{[25]}$ were first electropolymerized ${ }^{[26]}$ on the nanostructures arrays simply by applying $+0.57 \mathrm{~V}$ vs $\mathrm{Ag} / \mathrm{AgCl}$ in a solution containing NaDBS and pyrrole. ${ }^{[25]}$ This process was monitored by combined electrochemical and plasmonic sensing with high resolution. ${ }^{[27,28]}$ The polymer growth rate was linear ( $\sim 40 \mathrm{~nm} / \mathrm{min}$ dry thickness) and determined by integrating the electrochemical current (Figure S5, Supporting Information) and utilizing known film properties. ${ }^{[29]}$ After polymerization the metasurfaces absorb light at open circuit and the plasmons are damped. The color was switched "on" by applying approximately $-1 \mathrm{~V}(\mathrm{vs} \mathrm{Ag} / \mathrm{AgCl})$ in an electrolyte containing $0.1 \mathrm{M} \mathrm{NaDBS}$ and 0.1 $\mathrm{M} \mathrm{LiCl}$ in water (Figure 2a) to reduce the conductive polymer to its neutral state, thus altering its bandgap, ${ }^{[14,29]}$ leading to strongly reduced absorption. ${ }^{[24]}$ As a result the reflections from the RGB samples could be modulated with high contrast (spectra in Figure 2b). Note that the spectra are slightly shifted compared with Figure 1e since the structures now are in contact with polymer and electrolyte, but the colors appear very similar (photos in Figure 2b). For the shortest wavelengths $(<450 \mathrm{~nm}$ ) the blue and green structures actually showed reversed optical contrast. However, the human eye is not sensitive in this region under well-lit conditions where reflective displays are to be used. The wavelength interval where the luminosity function for photoptic vision is $>5 \%$ of its maximum is marked in Figure 2b.

An example of reflectivity vs time during potential switching is shown in Figure 2c for a red sample at the wavelength of $660 \mathrm{~nm}$ together with the electrochemical current. The response time, defined as the time to reach $90 \%$ of the intensity change, is on the order of hundreds of milliseconds depending on polypyrrole thickness ${ }^{[30]}$ (more detailed data in Supporting Information). This makes the technology suitable for reading devices although not high frame 


\section{Submitted to

rate animations, similarly to black and white electrophoretic displays. ${ }^{[12]}$ The switching was highly reproducible over long time as expected. ${ }^{[30,31]}$ After thousands of cycles the reduction in intensity change was $<10 \%$ without any special precaution to ensure a stable chemical environment (such as removing oxygen or switching to non-aqueous electrolytes).

Another important factor to consider is power consumption. As expected, ${ }^{[24]}$ the optical absorption did not simply scale linearly with the applied voltage (Figure S8, Supporting Information) and was also wavelength dependent. Considering further the stability of the electrochemical system, ${ }^{[27]}$ the ideal voltages for switching the colors fully on were $-0.9 \mathrm{~V}$, 1.0 V and -0.8 V for RGB respectively. Figure 2d shows the corresponding power densities for a steady “on” state determined from the electrochemical currents. Because of the low voltages and the thin polymer films, the power density is more than one order of magnitude lower compared to emissive displays and also much less than that for electrophoretic displays. ${ }^{[32]}$ The current trace (Figure $2 \mathrm{c}$ ) showed that switching the pixels every $\sim 10 \mathrm{~s}$ increased power density by less than a factor of three. Further, considering that not all pixels would be fully active (a white image) at a given time and that the polymer has coloration memory, ${ }^{[14]}$ the power consumption of the plasmonic metasurfaces is actually overestimated in Figure 2d (further details in Supporting Information).

In order to show that electronic paper based on the metasurfaces can be used to produce secondary colors and clear color images through RGB coding, we fabricated microscale pixels of the red, green and blue metasurfaces by three laser lithography steps and alumina depositions. The gold nanohole array was then prepared over the entire area in one step.

Figure 3a shows an example with $50 \mu \mathrm{m}$ pixels (approximately the resolution of human eyes) but the lithography can also produce pixels that are just a few microns, which corresponds to enough (tens of) nanoholes to excite surface plasmons, meaning that the maximum resolution is at least $10^{4}$ dpi. The photo in Figure 3a shows how the secondary colors yellow, purple and 


\section{Submitted to \\ ADNANCES
MAAERTALIS}

cyan can be produced by their RGB combinations. After placing the sample in an electrochemical cell and growing polypyrrole ( $200 \mathrm{~nm})$, the colors can be switched on and off over the entire area. Here we also refer to two videos available as Supporting Information, which shows the optical switching in real-time. One video simply shows switching of the whole sample area while the other shows preliminary results on polymer growth and switching of individual $100 \times 50 \mu \mathrm{m}$ pixels in a RGB triplet.

In order to quantitatively characterize the visual appearance of the plasmonic metasurfaces in relation to a known standard, we measured reflection spectra from ink spots produced by a laserjet printer on ordinary paper (Figure 3b). The printed paper thus acts as a "reflectivity standard" when designing the electronic paper. Assuming the electronic paper area will consist of 33\% red, 33\% green and 33\% blue pixels, the spectra from Figure 2b were normalized accordingly when compared with the reflectivity measured from the ink. We found that for optimal contrast a different polypyrrole thickness should be used for each color, namely 190 nm, $260 \mathrm{~nm}$ and $110 \mathrm{~nm}$ for RGB respectively. These are the values of dry polymer thickness for the data in Figure 3b (as well as previously in Figure 2b). Indeed, as illustrated by the arrows in Figure 3b, for all colors both the absolute reflectivity and the contrast are very similar to the printed paper, for which the "off” state is represented by black ink. This shows that electronic paper based on the plasmonic metasurfaces clearly has very similar visual appearance as standard color printed images. We also performed a chromaticity calculation to analyze the RGB colors according to the "standard observer" defined by the International Commission on Illumination ${ }^{[33]}$ (Figure S17, Supporting Information). As a final result, to show an image produced with the metasurface pixels, we patterned our university logo pixelated in RGB with different colors in different regions (photo in Figure 3c).

In summary we have presented the combination of conjugated polymers and plasmonic metasurfaces for reflective color displays, which we believe is the first demonstration of a 
olor. For future work, the next step is naturally to implement voltage control over the pixels individually. Notably, Figure 3a (and the online video) show non-optimal systems because the same polymer thickness and voltage is used for all colors. Further improvements are also possible by evaluating different materials and polymers for the different colors in order to improve contrast and response time. Also, power consumption can be further reduced in other electrolytes ${ }^{[31]}$ by lowering leakage currents and the energy required to maintain the polymer in its reduced state. To finalize the construction, a transparent flexible conductor such as graphene or indium-tin-oxide covered plastics should be used as counter electrode opposite to the metasurface, maintaining a thin electrolyte layer in between. It should be possible to follow the same design as for prototypes of flexible black and white electronic paper. ${ }^{[34]}$ This kind of reflective color displays should be especially useful for large-area energy-efficient electronic readers and posters which are also simple to fold and transport.

\section{Experimental Section}

Nanofabrication: The $0.5 \mathrm{~mm}$ thin glass slides were cleaned by $50 \mathrm{~W}$ oxygen plasma for 10 min. PET supports were cleaned by sonication in ethanol for $5 \mathrm{~min}$. All depositions were achieved with automated electron beam evaporation (Lesker PVD225). Underneath Ag a $5 \mathrm{~nm}$ Cr adhesion layer was used. Colloidal lithography was performed on freshly deposited $\mathrm{Al}_{2} \mathrm{O}_{3}$ as described previously ${ }^{[19,22,27]}$ using a batch of $158 \pm 4 \mathrm{~nm}$ colloids (Microparticles $\mathrm{GmbH}$ ), which give a short-range ordered pattern with characteristic spacing of $\sim 320 \mathrm{~nm}^{[22]}$ followed by deposition of $20 \mathrm{~nm}$ Au (with $1 \mathrm{~nm}$ Cr adhesion) and tape stripping to remove the colloids. ${ }^{[18]}$ Microscale pixels were patterned after Ag deposition by a laser writer (Heidelberg Instruments DWL 2000). The photoresist (Microposit S1813) was spin coated at $4000 \mathrm{rpm}$ and baked on a hotplate at $120^{\circ} \mathrm{C}$ for $2 \mathrm{~min}$. Pixels for one primary color were then patterned by the $60 \mathrm{~mW}$ laser beam after which the sample was developed in developer MF-318 for $50 \mathrm{~s} . \mathrm{Al}_{2} \mathrm{O}_{3}$ was 
then deposited and the process was repeated for each primary color. The gold nanohole array was then fabricated as for the other samples over the whole area in one step.

Spectroelectrochemistry: The dark spectrum of the spectrometer (B\&WTek CypherX) was recorded with the illumination off and subtracted from subsequent acquisitions. The light source was a $100 \mathrm{~W}$ halogen lamp (Newport). The reference intensity was measured as the reflection from a commercial Ag mirror (Thorlabs BBE02-E02). For measurements in the electrochemical cell the additional reflections (e.g. the opposite window) only caused a change in reflectivity of a few percent and were ignored. Reflectivity from metasurfaces was measured with a $10 \times$ air objective while for printed ink a 40× air objective was used to capture more of the diffuse reflection from the rough paper surface. High angle specular reflection was measured by a spectroscopic ellipsometer (Wollam M2000), which was also used to determine the complex refractive index of the dry polypyrrole. Extinction spectroscopy on ordinary nanohole arrays (Supporting Information) was performed as described previously. ${ }^{[27,28]}$ Note that reflectivity is the ratio of measured intensity and reference intensity, while extinction is the logarithm of the inverse of this ratio. A home-built liquid spectroelectrochemistry cell with chloridized Ag as reference electrode and Pt as counter electrode ${ }^{[27]}$ was connected to the potentiostat (Gamry Interface1000). The open circuit potential between our Ag electrode and a commercial Ag/AgCl standard (World Precision Instruments Dri-Ref) was measured before the experiments (typical difference $+80 \mathrm{mV}$ ) and the applied potential was adjusted accordingly. Electropolymerization was performed in $0.1 \mathrm{M}$ NaDBS (TCI Chemical, Japan) and $0.1 \mathrm{M}$ pyrrole (Sigma). For optical switching, 0.1 M LiCl (Sigma) was also added, mainly to improve reference electrode stability (although $\mathrm{Li}^{+}$ions also enter the polypyrrole layer just like $\mathrm{Na}^{+}$).

Photos: Pictures were taken with an Iphone 6 using standard settings, no flash and high dynamic range off. The microscope images were taken with a Thorlabs DCC1645C (CMOS) camera using standard settings. No parts of the photos have been manipulated. In all images that 


\section{Submitted to

compare "color on” and "color off” states, image settings such as brightness and contrast are identical.

Printing: The three primary colors and "black" were drawn in Paint on Windows 7. The red (R: 237, G: 28, B: 36) color was Hue 238, Sat 205, Lum 125, green (R: 34, G: 177, B: 76) was Hue 92, Sat 163, Lum 99 and blue (R: 0, G: 128, B: 255) was Hue 140, Sat 240, Lum 120. The printer was from HP (Laser Jet Pro 400 color MFP M475dn) and also the ink cartridge (Pink: CE413, Yellow: CE412, Cyan: CE411, Black: CE410). Ordinary white A4 paper was used (Future Multitech). The printing was done with "standard” quality settings in Windows.

\section{Supporting Information}

Supporting Information is available online from the Wiley Online Library or from the author.

\section{Acknowledgements}

This work was funded by the Swedish Research Council, the Swedish Foundation for Strategic Research and the Chalmers Nanoscience and Nanotechnology Area of Advance. We thank Prof. Olle Inganäs and Dr. Magnus Jonsson at Linköping University for helpful discussions regarding organic electronics.

Received: ((will be filled in by the editorial staff))

Revised: ((will be filled in by the editorial staff)) Published online: ((will be filled in by the editorial staff))

[1] I. Freestone, N. Meeks, M. Sax, C. Higgitt, Gold Bull. 2007, 40, 270.

[2] Y. H. Gu, L. Zhang, J. K. W. Yang, S. P. Yeo, C. W. Qiu, Nanoscale 2015, 7, 6409.

[3] T. D. James, P. Mulvaney, A. Roberts, Nano Lett. 2016, 16, 3817.

[4] T. Chen, B. M. Reinhard, Adv. Mater. 2016, 28, 3522.

[5] S. J. Tan, L. Zhang, D. Zhu, X. M. Goh, Y. M. Wang, K. Kumar, C. W. Qiu, J. K. W. Yang, Nano Lett. 2014, 14, 4023.

[6] J. C. Xue, Z. K. Zhou, Z. Q. Wei, R. B. Su, J. Lai, J. T. Li, C. Li, T. W. Zhang, X. H. Wang, Nat. Commun. 2015, 6, 8906.

[7] X. Zhu, C. Vannahme, E. Hojlund-Nielsen, N. A. Mortensen, A. Kristensen, Nat. Nanotechnol. 2016, 11, 325.

[8] Q. K. Liu, Y. Yuan, I. I. Smalyukh, Nano Lett. 2014, 14, 4071.

[9] D. Franklin, Y. Chen, A. Vazquez-Guardado, S. Modak, J. Boroumand, D. Xu, S.-T. Wu, D. Chanda, Nat. Commun. 2015, 6, 7337.

[10] T. Xu, E. C. Walter, A. Agrawal, C. Bohn, J. Velmurugan, W. Zhu, H. J. Lezec, A. A. Talin, Nat. Commun. 2016, 7, 10479.

[11] J. Olson, A. Manjavacas, T. Basu, D. Huang, A. E. Schlather, B. Zheng, N. J. Halas, P. Nordlander, S. Link, ACS Nano 2016, 10, 1108.

[12] P. F. Bai, R. A. Hayes, M. L. Jin, L. L. Shui, Z. C. Yi, L. Wang, X. Zhang, G. F. Zhou, Prog. Electromag. Res. 2014, 147, 95.

[13] B. Comiskey, J. D. Albert, H. Yoshizawa, J. Jacobson, Nature 1998, 394, 253. 
[14] P. Camurlu, RSC Adv. 2014, 4, 55832.

Submitted to

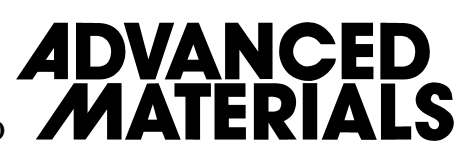

[15] Y. R. Leroux, J. C. Lacroix, K. I. Chane-Ching, C. Fave, N. Felidj, G. Levi, J. Aubard, J. R. Krenn, A. Hohenau, J. Am. Chem. Soc. 2005, 127, 16022.

[16] N. N. Jiang, L. Shao, J. F. Wang, Adv. Mater. 2014, 26, 3282.

[17] N. Yu, F. Capasso, Nat. Mater. 2014, 13, 139.

[18] J. Prikulis, P. Hanarp, L. Olofsson, D. Sutherland, M. Kall, Nano Lett. 2004, 4, 1003.

[19] J. Junesch, T. Sannomiya, A. B. Dahlin, ACS Nano 2012, 6, 10405.

[20] R. Ogier, L. Shao, M. Svedendahl, M. Kall, Adv. Mater. 2016, 28, DOI:

10.1002/adma.201600112.

[21] P. B. Johnson, R. W. Christy, Phys. Rev. B 1972, 6, 4370.

[22] A. B. Dahlin, M. Mapar, K. L. Xiong, F. Mazzotta, F. Hook, T. Sannomiya, Adv. Opt. Mater. 2014, 2, 556.

[23] T. Sannomiya, O. Scholder, K. Jefimovs, C. Hafner, A. B. Dahlin, Small 2011, 7, 1653.

[24] J. C. Gustafsson, O. Inganas, A. M. Andersson, Synth. Met. 1994, 62, 17.

[25] E. Smela, N. Gadegaard, Adv. Mater. 1999, 11, 953.

[26] S. Sadki, P. Schottland, N. Brodie, G. Sabouraud, Chem. Soc. Rev. 2000, 29, 283.

[27] A. B. Dahlin, R. Zahn, J. Voros, Nanoscale 2012, 4, 2339.

[28] A. B. Dahlin, J. O. Tegenfeldt, F. Hook, Anal. Chem. 2006, 78, 4416.

[29] E. Smela, M. Kallenbach, J. Holdenried, J. Microelectromech. S. 1999, 8, 373.

[30] D. Melling, S. Wilson, E. W. H. Jager, Smart Mater. Struct. 2013, 22, 104021.

[31] W. Lu, A. G. Fadeev, B. H. Qi, E. Smela, B. R. Mattes, J. Ding, G. M. Spinks, J. Mazurkiewicz, D. Z. Zhou, G. G. Wallace, D. R. MacFarlane, S. A. Forsyth, M. Forsyth, Science 2002, 297, 983.

[32] M. R. Fernandez, E. Z. Casanova, I. G. Alonso, Sustainability 2015, 7, 10854.

[33] T. Smith, J. Guild, Trans. Opt. Soc. 1931, 33, 73.

[34] R. Sakurai, S. Ohno, S.-I. Kita, Y. Masuda, R. Hattori, SID Symp. Digest Tech. Papers 2006, 37, 1922. 


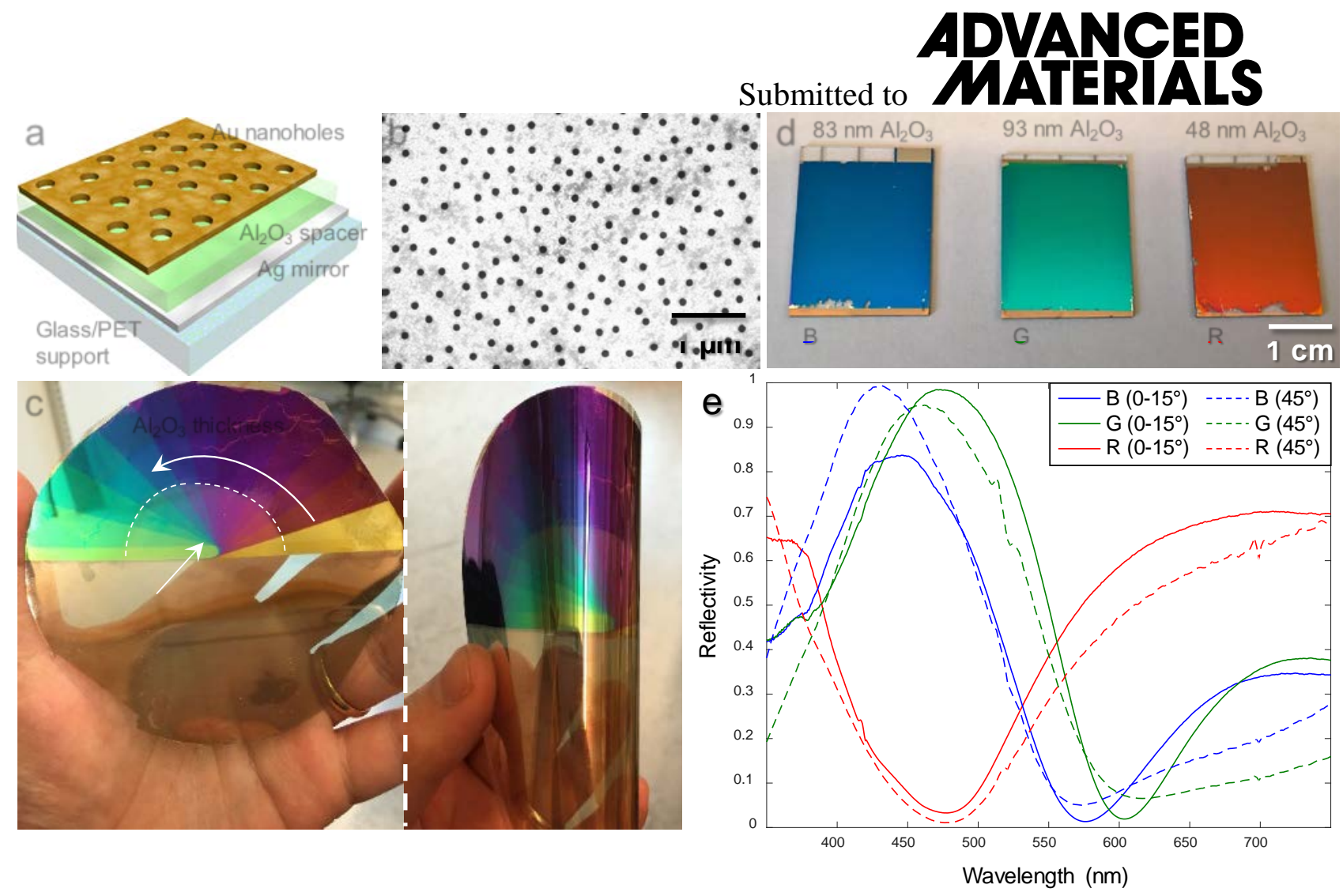

Figure 1. The plasmonic metasurfaces. a) Schematic of the metasurface. b) Electron microscopy image of the nanoholes from above. c) A photo of a sample on polyethylene terephthalate (PET) support with a color palette generated by a gradient in the alumina thickness. The middle region has no nanoholes in the gold film. d) A photo of samples with the primary colors under ambient light. e) Reflection spectra in air of the red, green and blue samples for different angles. 


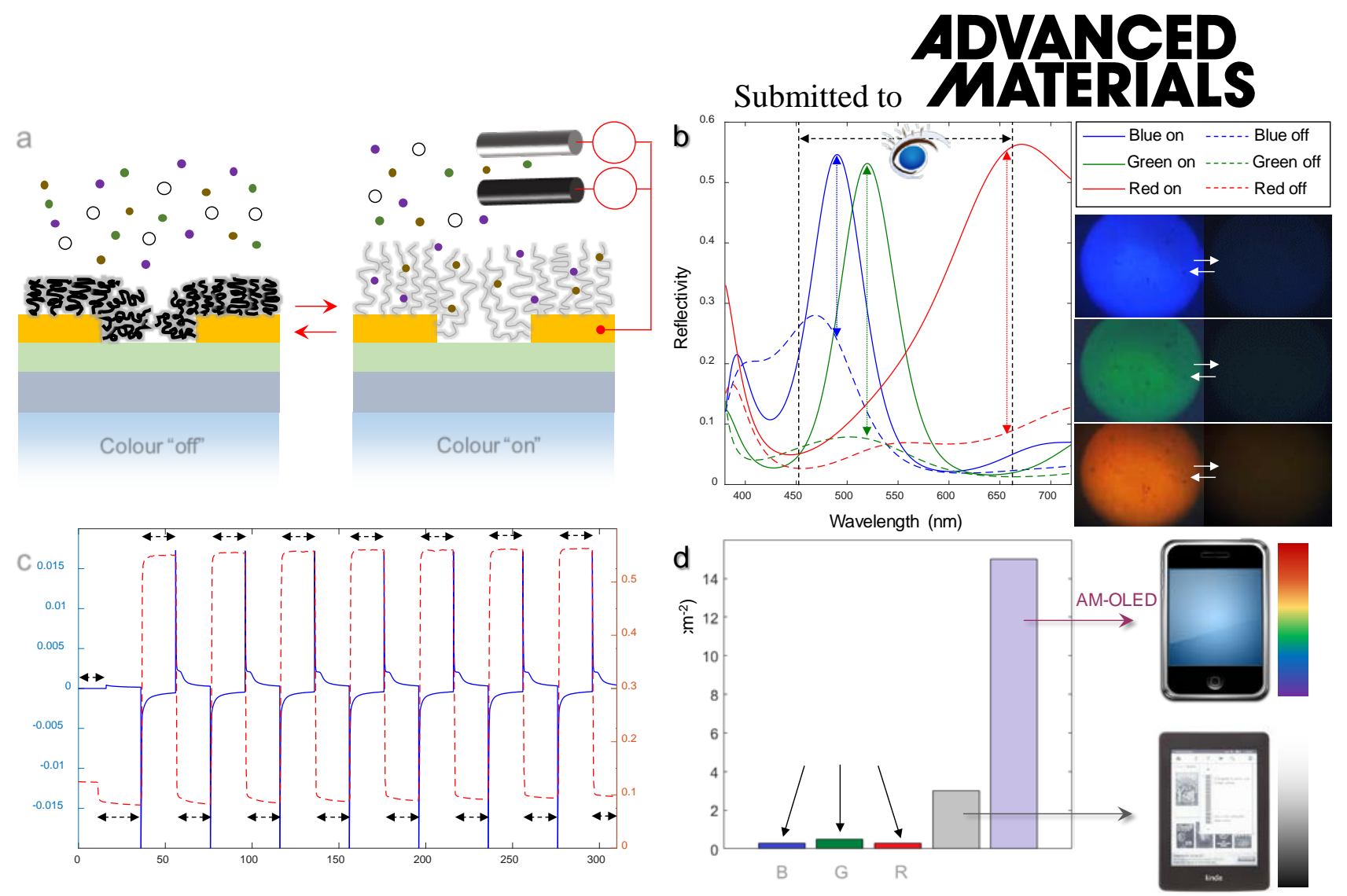

Figure 2. a) Principle of reflection modulation. After polypyrrole (PPy) has been electropolymerized on the gold nanohole array its optical absorption can be tuned by applying a potential as illustrated. b) The reflectivity in the bright and dark states for red, green and blue samples ( $0-14^{\circ}$ viewing angle). The dashed lines show the region where the luminosity function for photoptic vision is $>5 \%$ of its maximum. Also shown are microscope camera images of the samples ( $\sim 1 \mathrm{~mm}$ illuminated spot) in their bright and dark states (same illumination and brightness/contrast). c) Switching dynamics (for a red sample) showing current (electrode area $176 \mathrm{~mm}^{2}$ ) and reflectivity at $660 \mathrm{~nm}$ vs time as the voltage is reversed ( $>90 \%$ of the reflectivity change occurs in $<0.5 \mathrm{~s}$ ). d) The power density of the polymerfunctionalized plasmonic metasurfaces for the RGB colors compared with average power use for black and white electrophoretic displays (EPD) and AM-OLED displays. 


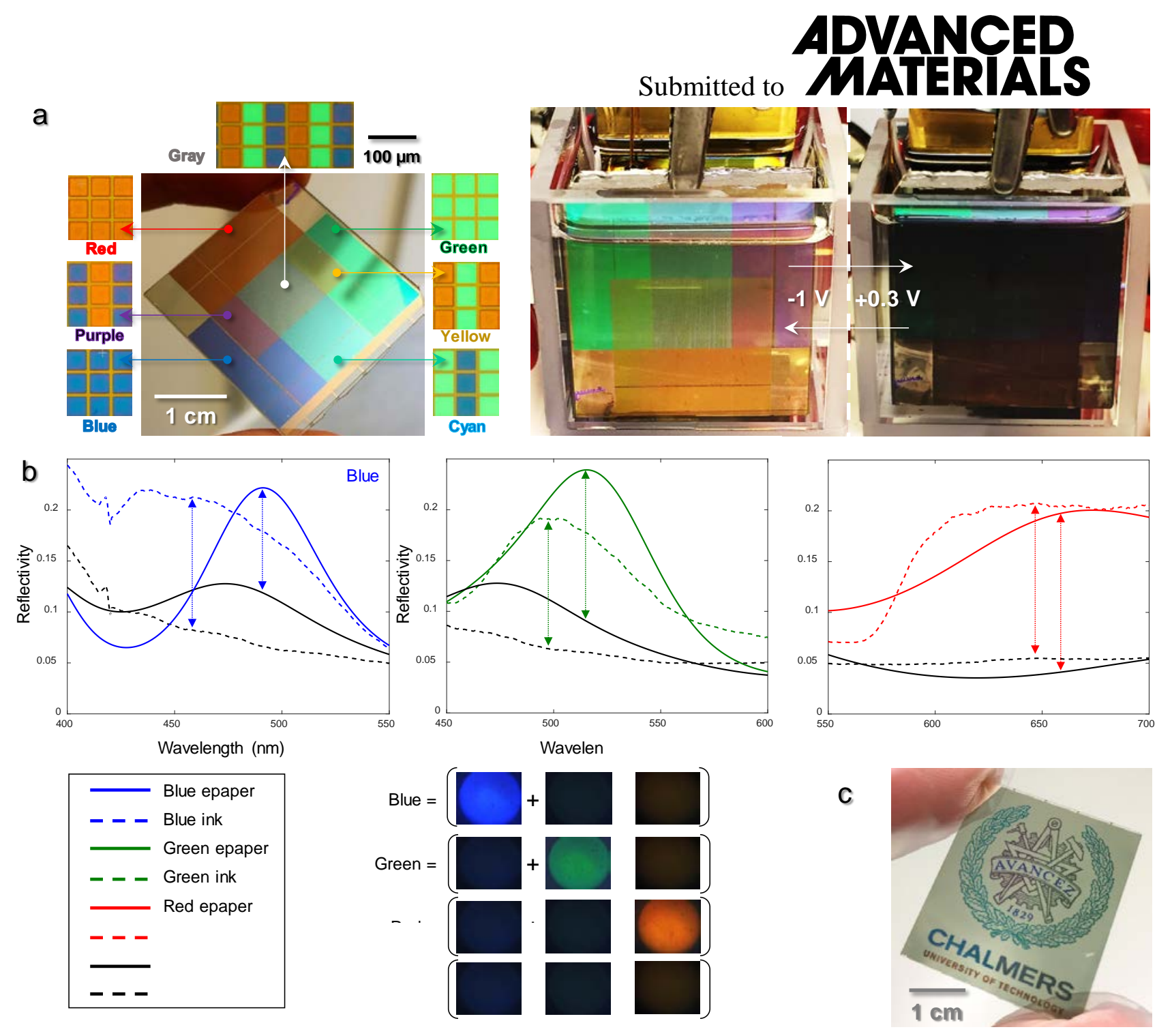

Figure 3. a) A photo of a sample with RGB pixels patterned to generate secondary colors. Also shown are photos of the same sample in an electrochemical cell after polymerization, with different voltages applied. b) Reflectivity comparison with printed ink. For each primary color, the reflectivity spectra (Figure 2b) were normalized by including the dark states for the other two colors, representing RGB coding with equal weights. The "off" state was defined as the average of the three dark states. For each color the spectral contrast was compared with the reflection measured from red, green, blue and black ink spots produced by a laserjet printer. c) A sample with RGB pixels patterned to display the university logo in different colors. 
ToC entry:

Flexible electronic paper in full color realized by plasmonic metasurfaces with conjugated polymers. An ultrathin large-area electrochromic material is presented which provides high polarization-independent reflection, strong contrast, fast response time and long-term stability. This technology opens up for new electronic readers and posters with ultralow power consumption.

plasmons, metasurfaces, conjugated polymers, electrochromism, electronic paper

K. Xiong, G. Emilsson, A. Maziz, X. Yang L. Shao, E.W.H. Jager and A.B. Dahlin.*

Plasmonic Metasurfaces with Conjugated Polymers for Flexible Electronic Paper in

Color

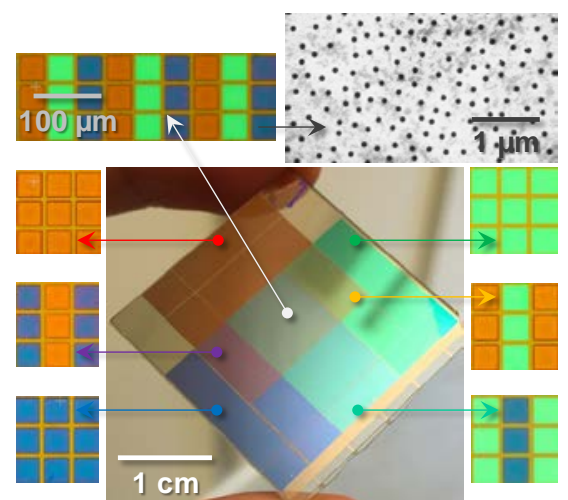

ToC figure 